\title{
A IMPORTÂNCIA DA HIDROTERAPIA EM PACIENTES ADULTOS COM ACIDENTE VASCULAR CEREBRAL
}

\author{
THE IMPORTANCE OF HYDROTHERAPY IN ADULT PATIENTS WITH STROKE \\ Tiago Neves de Santana \\ Centro Universitário Estácio do Recife
}

\begin{abstract}
The objective of this study was to evaluate the importance of hydrotherapy in the rehabilitation of adult patients affected by stroke. An integrative review of the literature using published scientific productions between the years 2011 to 2018 in the LILACS, Scielo and Bireme databases was carried out, using the descriptors: Stroke, Hydrotherapy and Physiotherapy. The results showed a significant importance of hydrotherapy in rehabilitation, with improvement of functional capacity, balance, gait, aerobic capacity and quality of life in adult patients with stroke. Thus, aquatic physiotherapy was shown to be very relevant and effective in patients suffering from stroke, both in rehabilitation and in the prophylaxis of new accidents.
\end{abstract}

Keywords: Stroke, Hydrotherapy and Physiotherapy.

\section{Resumo}

O objetivo desse estudo foi avaliar a importância da hidroterapia na reabilitação de pacientes adultos acometidos pelo acidente vascular cerebral (AVC). Foi realizada uma revisão integrativa da literatura utilizando produções científicas publicadas, entre os anos de 2011 a 2018, nas bases de dados LILACS, Scielo e Bireme, por meio dos descritores: Acidente Vascular Cerebral, Hidroterapia e Fisioterapia. Os resultados obtidos evidenciaram significativa importância da hidroterapia na reabilitação, com a melhora da capacidade funcional, do equilíbrio, da marcha, da capacidade aeróbica e da qualidade de vida em pacientes adultos com AVC. Assim, a fisioterapia aquática mostrou-se bastante relevante e eficaz em pacientes que sofreram AVC, atuando tanto na reabilitação, quanto na profilaxia de novos acidentes.

Palavras-chave: Acidente Vascular Cerebral, Hidroterapia e Fisioterapia. 


\section{Introdução}

O Acidente Vascular Cerebral (AVC) é uma das principais causas de incapacidade e morte no ocidente e, em especial, no Brasil. Trata-se de uma interrupção súbita do suprimento de sangue ao cérebro podendo ser devido a uma oclusão vascular (AVC isquêmico) ou a uma ruptura de um vaso (AVC hemorrágico) ${ }^{1,2}$.

Os fatores de risco mais importantes para o surgimento da doença vascular cerebral são a alta ingestão calórica e de gorduras, tabagismo, sedentarismo, hipertensão, hipercolesterolemia, Diabetes Mellitus e a idade ${ }^{3}$.

Os principais comprometimentos são tanto a nível motor, sensorial e auditivo, quanto no equilíbrio, na marcha, na fala e na área cognitiva. Esses efeitos vão depender do local e da extensão da lesão, da idade, do nível de condicionamento físico antes da lesão, se houve doenças associadas, e do tratamento recebido tanto na fase aguda quanto na fase tardia ${ }^{4,5}$.

Uma das principais alterações clínicas do AVC é a hemiplegia, quando há um déficit das funções motoras do hemicorpo contralateral à lesão encefálica. Geralmente, o padrão adotado pelo hemiplégico é de flexão do membro superior e extensão do membro inferior, havendo também outros padrões. Essas alterações levarão, consequentemente, a um déficit na independência, na capacidade funcional e na qualidade de vida do indivíduo ${ }^{2,6}$.

Dentre os vários métodos utilizados na fisioterapia para a reabilitação de pacientes acometidos pelo AVC, a hidroterapia vem se destacando e se firmando como um importante recurso para a reabilitação da capacidade funcional desses indivíduos ${ }^{7,8}$.

A água como terapia já vem sendo usada, por vários povos, há muitos anos para o tratamento de algumas doenças através dos banhos de imersão9. A ação terapêutica da água aquecida (na variância de 30 a $34^{\circ} \mathrm{C}$ ) otimiza o metabolismo, reduz a tensão e a dor muscular, além de promover um aumento temporário, após a imersão, dos níveis de dopamina ${ }^{10,11}$.

Dessa forma, a terapia aquática utiliza as propriedades da água como a temperatura, a viscosidade, o empuxo, a pressão hidrostática e o fluxo (coeficiente de arrasto) na reabilitação e na prevenção das desordens funcionais, para que esses ganhos possam ser passados para o solo e adicionados às atividades de vida diária dos indivíduos com $\mathrm{AVC}^{7,10,11}$.

Diante do alto índice de pessoas acometidas por AVC e a crescente utilização, nesses casos, da fisioterapia aquática como recurso terapêutico, esse trabalho tem por objetivo avaliar, através de uma revisão da literatura, a importância da hidroterapia na reabilitação de pacientes adultos acometidos pelo AVC.

\section{Metodologia}

Este estudo científico compreende uma revisão integrativa, a qual fundamenta-se numa ampla análise da literatura, a partir de uma questão ou tema delimitado, para sintetizar o que há de evidência científica acerca do assunto pesquisado ${ }^{12}$. Para a estruturação desta revisão, foram percorridas as seguintes etapas metodológicas: estabelecimento da questão norteadora; critérios de inclusão e exclusão dos artigos; definição das informações serem extraídas, a partir dos textos selecionados; interpretação e discussão dos resultados ${ }^{12}$. A pergunta norteadora foi: qual a relevância da hidroterapia na reabilitação de pacientes adultos com AVC?

Os critérios de inclusão definidos foram: estudos publicados entre 2011 a 2018 na língua portuguesa ou na inglesa; ser artigo original; com disponibilidade do texto completo nas bases de dados selecionadas. Foram excluídos: artigos repetidos, livros, teses, dissertações e artigos que não estivessem disponibilidade online gratuita.

A etapa seguinte foi constituída pela busca e seleção das produções científicas publicadas nas bases de dados LILACS, Scielo e Bireme, por meio dos Descritores em Ciências da Saúde (DeCS): Acidente Vascular Cerebral (Stroke), Hidroterapia (Hydrotherapy) e Fisioterapia (Physiotherapy), no período de busca de setembro de 2017 a agosto de 2018. Para proporcionar uma maior delimitação na busca, foi realizada uma intercessão entre os DeCS com o operador booleano "AND".

Inicialmente foram encontradas 21 publicações. Após uma leitura criteriosa, foram selecionadas apenas as que faziam correlações diretas entre a hidroterapia e pacientes acometidos pelo AVC, contabilizando um total de 8 artigos.

Logo em seguida, foi feita uma leitura explorativa e analítica, identificando o objeto, os 
objetivos e os resultados condizentes a cada pesquisa. Por se tratar de uma revisão integrativa, o estudo não foi submetido a um Comitê de Ética em Pesquisa.

\section{Resultados e discussões}

Diante dos artigos inclusos na pesquisa bibliográfica, observou-se que as técnicas hidroterápicas foram aplicadas em pacientes pósAVC, com tempo de pesquisa variando de 8 a 31 sessões, com duração mínima de 35 e máxima de 60 minutos. As variáveis analisadas nas pesquisas foram a descarga de peso, tônus muscular, equilíbrio, funcionalidade, marcha, condicionamento cardiovascular e a qualidade de vida (tabela 1).

Tabela 1. Resultados da aplicação da hidroterapia em pacientes pós-AVC.

\begin{tabular}{|c|c|c|c|c|}
\hline Técnicas & $\begin{array}{l}\text { Tempo } \\
\text { Pesquisa }\end{array}$ & Intercorrências & Resultados & Autor(es)/Ano \\
\hline Bad Ragaz & $\begin{array}{l}24 \text { sessões de } \\
45 \text { minutos }\end{array}$ & $\begin{array}{l}\text { AVC } \\
\text { Hemorrágico }\end{array}$ & $\begin{array}{l}\text { Melhora da função, do equilíbrio e na } \\
\text { qualidade de vida; } \\
\text { Não houve alteração do tônus. }\end{array}$ & $\begin{array}{l}\text { CARVALHO, } \\
\text { BASSI. } 2017\end{array}$ \\
\hline Hidroterapia & $\begin{array}{l}31 \text { sessões de } \\
60 \text { minutos }\end{array}$ & AVC & Melhora do condicionamento físico. & $\begin{array}{l}\text { JAKAITIS et al. } \\
2011\end{array}$ \\
\hline Hidroterapia & $\begin{array}{l}8 \text { sessões de } \\
35 \text { minutos }\end{array}$ & AVC Isquêmico & $\begin{array}{l}\text { Melhora na descarga de peso no } \\
\text { hemicorpo parético. }\end{array}$ & LIMA et al. 2013 \\
\hline Halliwick & $\begin{array}{l}24 \text { sessões de } \\
50 \text { minutos }\end{array}$ & AVC & $\begin{array}{l}\text { Melhora no equilíbrio e na } \\
\text { funcionalidade. }\end{array}$ & $\begin{array}{l}\text { MENEGHETTI et } \\
\text { al. } 2012\end{array}$ \\
\hline Halliwick & $\begin{array}{l}18 \text { sessões de } \\
40 \text { minutos }\end{array}$ & AVC & $\begin{array}{l}\text { Melhora do equilíbrio; Não houve } \\
\text { melhora significativa na qualidade de } \\
\text { vida. }\end{array}$ & $\begin{array}{l}\text { MONTAGNA et } \\
\text { al. } 2014\end{array}$ \\
\hline Hidroterapia & $\begin{array}{l}10 \text { sessões de } \\
50 \text { minutos }\end{array}$ & AVC & $\begin{array}{l}\text { Melhora no condicionamento } \\
\text { cardiovascular e na qualidade de vida. }\end{array}$ & $\begin{array}{l}\text { COSTA et al. } \\
2017\end{array}$ \\
\hline Hidroterapia & $\begin{array}{l}12 \text { sessões de } \\
60 \text { minutos }\end{array}$ & AVC & $\begin{array}{l}\text { Melhora na performance da } \\
\text { mobilidade funcional. }\end{array}$ & $\begin{array}{l}\text { SANTOS et al. } \\
2011\end{array}$ \\
\hline $\begin{array}{l}\text { Halliwick } \\
\text { Bad Ragaz }\end{array}$ & $\begin{array}{l}18 \text { sessões de } \\
45 \text { minutos }\end{array}$ & AVC & $\begin{array}{l}\text { Aumento da qualidade de vida, da } \\
\text { velocidade da marcha e da } \\
\text { capacidade funcional. }\end{array}$ & $\begin{array}{l}\text { TONIETO et al. } \\
2015\end{array}$ \\
\hline
\end{tabular}

Dentro dos critérios de inclusão e exclusão utilizados, foram encontrados oito artigos sendo sete escritos na língua portuguesa e um na língua inglesa. Em suma, foi observado que a hidroterapia promoveu melhora da função, do equilíbrio, da descarga de peso, do condicionamento cardiovascular, do tônus muscular, da marcha e da qualidade de vida dos pacientes com AVC.

Por ser um dos distúrbios circulatórios mais comuns em todo mundo, o AVC é alvo de vários estudos que buscam utilizar terapias eficazes na redução dos danos decorrentes e suas complicações. Pesquisas mostram que pacientes acometidos pelo AVC podem apresentar inúmeras alterações neurológicas correlacionadas com áreas importantes do corpo, como a motora, a cognição e a fala, podendo levar também a limitações nas atividades de vida diária do indivíduo ${ }^{1,3,5,13}$.
Então Lima et al. ${ }^{14}$ investigaram, em sua pesquisa, se a fisioterapia aquática era eficaz na transferência de peso no hemicorpo acometido durante a passagem de sentado para ortostatismo, através de uma paciente de 50 anos com diagnóstico de AVC isquêmico. Após 8 sessões de hidroterapia em piscina aquecida, obteve-se uma ativação mais positiva da musculatura do glúteo máximo favorecendo uma maior harmonia e simetria na passagem de sentado para a posição ortostática, além de uma descarga de peso mais equilibrada. Esses ganhos partem do princípio de que quando o corpo é imerso na água, a ação da gravidade vai sendo anulada pela atuação da flutuação, propiciando uma descarga de peso mais efetiva e gradual para o lado hemiparético do paciente, favorecendo uma ativação mais equilibrada dos músculos nos membros inferiores na posição ortostática $^{7,8,15}$. 
Avaliando a questão do equilíbrio e da qualidade de vida, Montagna et al. ${ }^{4}$ utilizaram pacientes com AVC crônico. Com a técnica hidroterápica Halliwick, os participantes obtiveram melhora tanto no equilíbrio estático (avaliado pela Escala de Equilíbrio de Berg), quanto no equilíbrio dinâmico (pelo teste Timed Up \& Go). Corroborando com os resultado obtidos por Meneghetti et al. ${ }^{16}$ que também utilizaram o Halliwick como técnica de tratamento e obtiveram melhora nas pontuações das escalas avaliativas de 15 para 40 pontos, referentes ao equilíbrio e, de 91 para 108 pontos, relativos à independência funcional ${ }^{16}$.

Essa melhora do equilíbrio e da independência funcional pode ser atribuída à utilização do Halliwick que segue o princípio do desprendimento tanto através de atividades aquáticas quanto de intervenções fisioterápicas ${ }^{4,16,17}$. Por meio da relação um-praum do fisioterapeuta com o paciente, usando pouco apoio, o mesmo aprende a controlar melhor o corpo na água, estimulando o equilíbrio sem ajuda dos flutuadores. E, após reaprendidas habilidades importantes como mudar ou manter a posição corporal controladamente, o paciente consegue responder com maior flexibilidade a situações e estímulos diferentes de forma mais eficiente e independente ${ }^{17}$.

Carvalho, Bassi ${ }^{18}$ também avaliaram os efeitos da hidroterapia no equilíbrio e na qualidade de vida em um estudo de caso. No entanto, a técnica utilizada para o tratamento foi - método dos anéis de Bad Ragaz que é composto por movimentos com padrões em planos anatômicos e diagonais, com a estabilização e a resistência dadas pelo fisioterapeuta. Constatou-se também uma melhora no equilíbrio e na qualidade de vida. No entanto, o resultado já não foi tão significativo tanto na funcionalidade quanto no tônus muscular quando comparado pré e pósintervenção.

De forma mais abrangente no tocante à hidroterapia como intervenção para melhora do equilíbrio, sabe-se que uma das propriedades físicas da água bastante explorada é a flutuação. Por meio dela é possível abordar a questão do equilíbrio estático e dinâmico, tanto trabalhando o paciente para mantê-lo relaxado e imóvel, quanto estimulando as reações de equilíbrio dinâmico diante de uma turbulência gerada na água através dos exercícios, permitindo assim uma maior consciência corporal e orientação espacial do paciente $e^{6,10,17}$.

Já com o objetivo de avaliar a capacidade funcional, a velocidade da marcha e a qualidade de vida, Tonieto et al. ${ }^{19}$ aplicaram a fisioterapia aquática em pacientes pós-AVC. Após as 18 sessões de reabilitação, obtiveram melhora significante na qualidade de vida, no condicionamento cardiovascular e na marcha dos pacientes avaliados. A melhora pode ser atribuída ao método Halliwick que, por meio da posição de cubos, favorece a manutenção da dorsiflexão e melhora do equilíbrio, e também ao método Bad Ragaz que associado às propriedades físicas da água como a turbulência e a viscosidade, favorece a ativação dos músculos abdominais e paravertebrais promovendo uma maior estabilidade do tronco, adequação do tônus muscular, melhora da amplitude das articulações, força muscular e serve como prétreinamento de marcha ${ }^{16,18,19}$.

Por conta da espasticidade, mostrou-se que em pacientes acometidos pelo AVC, há um déficit no controle motor voluntário gerando uma diminuição na capacidade de iniciar o movimento ${ }^{1,7}$. Então, um estudo foi realizado para avaliar a capacidade funcional de pacientes pós-AVC ${ }^{20}$. A partir do teste Timed Up and Go, a mobilidade funcional, o equilíbrio, a velocidade da marcha e a transferência de sentado para em pé foram avaliadas antes e após 12 sessões de hidroterapia. Quando compararam os resultados pré-teste da primeira avaliação e pós-teste da última avaliação, os dados mostraram uma diminuição no tempo do Timed Up and Go, em longo prazo, evidenciando uma melhora na performance da mobilidade funcional de todos os participantes ${ }^{20}$.

Esses ganhos positivos obtidos na funcionalidade do lado hemiparético são devidos à estimulação da hidroterapia, tanto pela temperatura da água, que vai reduzir a espasticidade e diminuir a dor, quanto pela flutuação que vai promover uma boa sustentação do corpo, facilitando a mobilização das articulações pelo terapeuta, e, posteriormente, a perpetuação da amplitude de movimento ganhada na água, gerando uma maior independência funcional do paciente acometido pelo $\mathrm{AVC}^{7,11}$.

Partindo da informação que a realização de atividade física pode ser comprometida por causa da alta demanda energética gerada pela 
disfunção motora decorrente do AVC, podendo levar a uma fadiga muscular precoce $\mathrm{e}$ dificultando o condicionamento desses pacientes ${ }^{21}$, Jakaitis et al. ${ }^{13}$ fizeram um trabalho analisando o condicionamento físico, assim como Costa et al. ${ }^{22}$ que avaliaram o condicionamento cardiovascular e também a qualidade de vida, após tratamento com a fisioterapia aquática. No final do primeiro estudo não houve alterações dos resultados da frequência cardíaca, do esforço e da pressão arterial, mostrando que a hidroterapia foi eficaz na melhora do condicionamento geral após os 6 meses de terapia. Corroborando com os resultados do segundo estudo, onde o grupo experimental também obteve melhora da condicionamento cardiovascular e da qualidade de vida.

Esses resultados positivos no condicionamento físico e cardiovascular devemse à pressão que a água exerce sobre cada parte do corpo que é chamada de pressão hidrostática. Essa pressão vai promover uma estimulação à nível periférico, melhorando a circulação e o retorno venoso. Também vai atuar, juntamente com a flutuação, gerando uma resistência natural para o fortalecimento da musculatura respiratória e melhorando o suprimento sanguíneo para os músculos durante os exercícios da hidroterapia ${ }^{9,11,15}$.

Estudos mostram que os exercícios em meio aquático promovem não só benefícios físicos mais também mentais e sociais ${ }^{17}$. As propriedades físicas da água promovem uma melhora da capacidade de movimento, da descarga de peso, da função, do tônus muscular, do equilíbrio e das capacidades cardiorrespiratórias, levando o paciente com AVC a sair de sentimentos de isolamento ou depressão e tornar-se mais independente e mais ativo socialmente, aumentando sua percepção da qualidade de vida ${ }^{10,11,17}$.

\section{Considerações finais}

De acordo com o presente estudo, concluise que a fisioterapia aquática mostrou-se bastante eficaz em pacientes que sofreram AVC, atuando na reabilitação motora (melhorando a capacidade funcional, o equilíbrio, a marcha, a capacidade aeróbica e a qualidade de vida), na reabilitação psicossocial (a partir do momento que o paciente começa a ter suas aquisições motoras restabelecidas, há um encorajamento para voltar ao seu âmbito social e suas atividades de vida diária), e na profilaxia de novos acidentes.

A relevância dessa pesquisa se encontra no fato dos resultados positivos encontrados utilizando a hidroterapia para o tratamento das desordens do AVC, possibilitando novos aprendizados sobre o tema, aumentando o acervo de conhecimentos dos fisioterapeutas para a prática clínica e servindo de base para futuras produções científicas. Vale destacar a escassez de estudos na área, o que limitou que os resultados fossem mais abrangentes e de forma mais equilibrada nos grupos estudados.

Com isso, sugere-se que novos estudos sejam desenvolvidos para que se amplie o campo de investigação e com intervenções mais abrangentes sobre a atuação da hidroterapia como método eficaz na reabilitação de indivíduos acometidos pelo AVC.

\section{Referências:}

1. Lima AP, Cardoso FB. O efeito de um programa de exercícios físicos sobre a capacidade funcional da marcha hemiparética de indivíduos com acidente vascular cerebral. Rev Bras Cien Saúde. 2014; 18(3):203-208.

2. Piassaroli CAP, Almeida GC, Luvizotto JC, Suzan ABBM. Modelos de reabilitação fisioterápica em pacientes adultos com sequelas de AVC Isquêmico. Rev Neuroc. 2011; 20(1):128137.

3. Zaleski TDP, Camera FD, Wisniewski E, Wisniewski MSW. Avaliação da força muscular respiratória e função pulmonar em indivíduos com acidente vascular cerebral. Rev Perspect. 2018; 42(edição especial):15-22.

4. Montagna JC, Santos BC, Battistuzzo CR, Loureiro APC. Effects of aquatic physiotherapy on the improvement of balance and corporal symmetry in stroke survivors. Intern Jour Clin and Exp Med. 2014; 7(4):1182-1187.

5. Pompeu JE, Alonso TH, Masson IB, Pompeu SMAA, Passin CT. Os efeitos da realidade virtual na reabilitação do acidente vascular encefálico: uma revisão sistemática. Rev Motric. 2014; 10(4):111-122.

6. Ferla FL, Grave M, Perico E. Fisioterapia no tratamento do controle de tronco e equilíbrio de pacientes pós AVC. Rev Neuroc. 2015; 23(2):211-217.

7. Baccaro VM, Kanashiro MS, Batista BP, Beas ARV, Souza CM, Moraes AL. Uso do 
Biofeedback na Hidroterapia em pacientes com acidente vascular encefálico. Saúde Rev. 2016; 16(42):47-56.

8. Coelho CCS, Lemos TSA, Luzes R. Os efeitos da hidroterapia na recuperação da amplitude de movimento. Rev Disc UNIABEU. 2015; 3(6):1-6.

9. Silva MR. Hidroterapia no tratamento da fibromialgia [monografia]. Ariquemes: Faculdade de Educação e Meio Ambiente; 2014.

10. Silva DM, Nunes COM, Oliveira PJAL, Coriolano MGWS, Berenguer FA, Lins OG, et al. Efeitos da fisioterapia aquática na qualidade de vida de sujeitos com doença de Parkinson. Fisio Pesq. 2013; 20(1):17-23.

11. Bastos VPD, Bezerra MVA, Vasconcelos TB, Câmara TMS, Sousa CT, Macena RHM. Benefícios da hidroterapia nos pacientes portadores de sequela de acidente vascular cerebral. Saúd Sant Mar - Suplem. 2016; 7-14.

12. Lelis $\mathrm{CM}$, Battaus MRB, Freitas FCT, Rocha FLR, Marziale MHP, Robazzi MLCC. Distúrbios osteomusculares relacionados ao trabalho em profissionais de enfermagem: revisão integrativa da literatura. Acta Paul Enferm. 2012;25(3):477-82.

13. Jakaitis F, Santos DG, Abrantes CV, Gusman S, Bifulco SC. Atuação da fisioterapia aquática no condicionamento físico do paciente com AVC. Rev Neuroc. 2011; 20(2):204-209.

14. Lima PN, Souza CM, Andrade SS, Cyrillo FN, Braga DM. Fisioterapia aquática na transferência do sentado para ortostatismo no paciente com AVC: relato de caso. Rev Neuroc. 2013; 21(2):251-257.

15. Cunha JVB, Martins HAP. Efeitos da terapia aquática sobre a dor lombar crônica: estudo de caso [monografia]. Lins: Centro Universitário Católico Salesiano Auxilium; 2016.

16. Meneghetti $\mathrm{CHZ}$, Carraro L, Leonello LA, Batistella ACT, Ferracini LCJ. A influência da fisioterapia aquática na função e equilíbrio no acidente vascular cerebral. Rev Neuroc. 2015; 20(3):410-414.

\section{Endereço para Correspondência}

Centro Universitário Estácio do Recife

Avenida Engenheiro Abdias de Carvalho, №:

1678. Recife - PE
CEP.: $58428-830$

e-mail: tiagonds@hotmail.com

Recebido em 22/11/2019

Aprovado em 13/04/2020

Publicado em 09/07/2020 